\title{
Topically applied ceramide accumulates in skin glyphs
}

This article was published in the following Dove Press journal:

Clinical, Cosmetic and Investigational Dermatology

I July 2015

Number of times this article has been viewed

\author{
Qihong Zhang' \\ Carol R Flach' \\ Richard Mendelsohn' \\ Guangru Mao² \\ Apostolos Pappas ${ }^{2}$ \\ M Catherine Mack ${ }^{2}$ \\ Russel MWalters ${ }^{2}$ \\ Michael D Southall ${ }^{2}$ \\ 'Department of Chemistry, Rutgers \\ University, Newark, 'Johnson \& \\ Johnson Consumer Companies, Inc., \\ Skillman, NJ, USA
}

\begin{abstract}
Ceramides (CERs), structural components of the stratum corneum (SC), impart essential barrier properties to this thin outer layer of the epidermis. Variations in CER species within this layer have been linked to several skin diseases. A recent proliferation of CERcontaining topical skin-care products warrants the elucidation of CER penetration profiles in both healthy and diseased skin. In the current study, the spatial distributions of CER concentration profiles, following topical application of two species of CER, were tracked using infrared imaging. Suspensions of single-chain perdeuterated sphingosine and phytosphingosine CER in oleic acid were applied, in separate experiments, to the surface of healthy intact ex vivo human skin using Franz diffusion cells. Following either a 24 - or 48 -hour incubation period at $34^{\circ} \mathrm{C}$, infrared images were acquired from microtomed skin sections. Both CER species accumulated in glyph regions of the skin and penetrated into the SC, to a limited extent, only in these regions. The concentration profiles observed herein were independent of the CER species and incubation time utilized in the study. As a result, a very heterogeneous, sparse, spatial distribution of CERs in the SC was revealed. In contrast, oleic acid was found to be fairly homogeneously distributed throughout the SC and viable epidermis, albeit at lower concentrations in the latter. A more uniform, lateral distribution of CERs in the SC would likely be important for barrier efficacy or enhancement.
\end{abstract}

Keywords: stratum corneum, infrared imaging, topical delivery, oleic acid

\section{Introduction}

The composition and molecular order of stratum corneum (SC) lipids are critical in the barrier function of skin. SC lipids are composed of approximately equimolar ratios of ceramides (CERs), cholesterol, and free fatty acids as well as small amounts of cholesterol esters and cholesterol sulfate. CERs play an essential structural role in the barrier properties of the epidermis. Besides their structural role in the SC, they also serve as intracellular signaling molecules in the viable epidermis (VE) and regulate several biological processes, such as proliferation, differentiation, apoptosis, inflammation, and immune responses. ${ }^{1}$

Variations in skin CERs have been linked to various skin conditions. Many disorders, including atopic dermatitis ${ }^{2,3}$ and psoriasis, ${ }^{4}$ often show an impaired barrier function and present either a decrease in total CER content or differences in the relative amounts of chemical species of CERs. Other skin diseases have also been linked to lipid composition and structural order abnormalities, including lamellar ichthyosis, Netherton and Chanarin-Dorfman. ${ }^{5}$

There has been a proliferation of topical skin-care products containing CERs intended for use by typical, healthy consumers. Despite limited in vitro or clinical
Correspondence: Carol R Flach Department of Chemistry, Rutgers University, 73 Warren Street Newark, NJ 07102, USA

Tel $+|973353| 330$

Fax +I 9733531264

Email flach@andromeda.rutgers.edu 
evidence utilizing healthy human skin, CERs have been included in hundreds of commercial skin-care products claiming to increase the skin's hydration level, repair the cutaneous barrier, prevent moisture loss, and contribute to reducing dry and flaky skin. Whereas many CER supplementation experiments conducted after acute barrier disruption of the SC demonstrated recovery, ${ }^{6} \mathrm{CER}$ penetration, and thus its course of action, is likely to differ in healthy skin. In a recent review, the conclusion was drawn that perturbed skin barrier recovery depends on applying the appropriate composition of SC lipids. ${ }^{7}$ It was further stated that the exogenous, physiological lipids are transported through the SC into the stratum granulosum (SG) cells. Two separate reports have appeared studying CER transport in uncompromised, ex vivo human skin. In the first, the penetration of fluorescently labeled CERs was shown to depend on acyl chain length, ${ }^{8}$ with fluorescence of short-chain (C6) CER observed in the VE and long chain (C24) CER observed only in the outer layers of the SC. In the second study, the permeability of a deuterated CER was shown to depend on the delivery system used. ${ }^{9}$ It is evident that additional studies are needed to probe CER penetration in healthy skin.

While endogenous skin CERs are clearly important in maintaining the skin barrier and regulating water flux through the skin, the effect of topically applied exogenous CER on the SC is unclear. Ideally, CER supplementation of skin would result in the topically applied CER diffusing throughout the SC and strengthening its barrier properties. The initial organization of SC lipids takes place at the SG-SC interface, following enzymatic hydrolysis of lipid precursors to the final SC lipid constituents, ${ }^{2,10,11}$ and suggesting that it is necessary to deliver the topically applied CER to the SG. However, it is difficult to administer the lipids deep into the interface in healthy skin using conventional formulations such as ointments and creams. ${ }^{12,13}$ As an alternative, formulations utilizing nanoemulsions, including solid lipid nanoparticles, for transdermal and dermal delivery of actives are being actively investigated. ${ }^{14-16}$

A limitation in studying CER penetration into skin has been the lack of sensitive instrumentation with the ability to differentiate exogenous CER from endogenous CER. Infrared (IR) imaging is a vibrational spectroscopy-based method, which provides molecular structure information about endogenous skin components in addition to tracking the exogenous agent whose penetration is being monitored. Over the past 20 years, the development of IR microspectroscopic imaging has proven the feasibility of acquiring spatially resolved spectra from tissue including microtomed skin sections. ${ }^{17-22}$
Penetration of exogenous agents is conveniently tracked from the spatial distribution of their vibrational band intensities. Although IR microscopic imaging has not been previously used, to the best of our knowledge, to measure CER penetration into skin, studies demonstrating the feasibility of the experiment have indeed appeared. Gotter et $\mathrm{al}^{23}$ investigated the heterogeneous lateral distribution of the anti-psoriasis drug dithranol in an artificial membrane. Mao et al ${ }^{19}$ imaged the distribution of sodium dodecyl sulfate across different skin regions. A useful advantage of IR imaging was realized from the fact that Beer's law is obeyed in the IR, thereby permitting (in the example cited) determination of the sodium dodecyl sulfate concentrations in the skin. Finally, IR microspectroscopic imaging has been used to investigate the penetration of oleic acid (OA) and glycerol trioleate in ex vivo human skin. ${ }^{24}$

The goal of the current study is to determine if topically applied CER penetrates and augments the lipids in the SC in ex vivo intact skin. We demonstrate the feasibility of mapping CER permeation in human skin with IR imaging. We imaged the penetration of topically applied deuterated CERs with a common permeation enhancer, OA. The use of single-chain perdeuterated N-palmitoyl-D-erythro-sphingosine (CER [NS]-d31) or N-palmitoyl-D-erythro-phytosphingosine (CER [NP]-d31) permits us to spectroscopically distinguish the exogenous agents from the endogenous SC lipids without affecting penetration parameters. The CERs chosen for this study have also been identified in the final report of the 2015 Cosmetic Ingredient Review Expert Panel ${ }^{25}$ as being among the top three CERs having the most reported uses in cosmetic and personal care products. The major use of these CERs is in leave-on skin-care preparations. In the study reported here, images of CER concentration were generated after applying extinction coefficients determined from Beer's law. The results demonstrate that $\mathrm{CER}$ applied with $\mathrm{OA}$ to intact skin remains predominantly on the surface of the $\mathrm{SC}$, suggesting that topical replenishment of CER may not be an effective approach to improve the barrier properties of healthy skin.

\section{Materials and methods Materials}

Acid-chain perdeuterated N-palmitoyl-D-erythro-sphingosine (CER [NS]-d31) and acid-chain perdeuterated N-palmitoylD-erythro-phytosphingosine (CER [NP]-d31) were purchased from Avanti Polar Lipids, Inc. (Alabaster, AL, USA). OA and acyl chain perdeuterated oleic acid (OA-d) were purchased from Sigma-Aldrich Co (St Louis, MO, USA). Human abdominal skin from plastic surgery procedures was 
obtained from several dermatological offices following informed consent and ethics board approval. Samples were fast frozen with liquid nitrogen after removal of subcutaneous fat, cut into approximately $15 \times 15 \mathrm{~cm}^{2}$ pieces, and stored at $-20^{\circ} \mathrm{C}$ for 6 months or less.

\section{Methods}

\section{Sample preparation}

CER was mixed with OA to make suspensions at $\sim 10-12$ $\mathrm{mg} / \mathrm{mL}$ of CER (approximately five times than that commonly found in commercial formulations). Skin samples of $\sim 2 \times 2 \mathrm{~cm}^{2}$ surface area and $\sim 2.5 \mathrm{~mm}$ thickness were cut from a larger section and defrosted on filter paper with the SC side up. The SC was cleaned with cotton swabs and tape-stripped twice to remove most of the sebum.

For the CER permeability study, skin samples were mounted and secured on a Franz diffusion cell (PermeGear, Inc., Hellertown, PA, USA) with the SC facing the donor compartment. The acceptor compartment was partially filled with distilled water, and, in separate experiments, $50 \mu \mathrm{L}$ of each suspension was applied on the skin surface $(\sim 0.64$ $\mathrm{cm}^{2}$ ). Samples, wrapped with Parafilm $\mathrm{M}^{\circledR}$ to minimize water evaporation and to keep skin hydrated, were incubated at $34^{\circ} \mathrm{C}$ for 24 or 48 hours in separate experiments. Control experiments were conducted under the same conditions with topical application of only OA-d or proteated CER suspensions in OA-d. After incubation, the excess suspension was removed with a cotton swab, the apparatus was disassembled, the skin surface thoroughly wiped with Kimwipes ${ }^{\mathrm{TM}}$, and the center part of the skin samples was fast frozen with liquid $\mathrm{N}_{2}$.

\section{IR microscopic imaging}

For IR imaging, samples prepared as described in the previous subsection were stabilized using a drop of water without embedding agents at $-30^{\circ} \mathrm{C}$ within a Bright/Hacker 5030 Microtome (Bright Instrument Co Ltd, Huntingdon, UK; Hacker Instruments Inc., Fairfield, NJ, USA). Sections ( $\sim 10 \mu \mathrm{m}$ thick) were cut perpendicular to the SC surface and placed on $\mathrm{CaF}_{2}$ IR windows. IR images were collected with a PerkinElmer Spotlight 300 system (PerkinElmer Inc., Waltham, MA, USA) in transmission mode with an essentially linear array $(16 \times 1)$ of mercury-cadmium-telluride detector elements. A $6.25 \times 6.25 \mu^{2}$ pixel size was utilized and 32 scans were acquired at a spectral resolution of $8 \mathrm{~cm}^{-1}$. Image sizes were $\sim 500 \times 200 \mu^{2}$, corresponding to 80 pixels in the $\mathrm{x}$ direction and 32 pixels in the $\mathrm{y}$ direction. Visible micrographs were obtained with the microscope integrated into the Spotlight 300 system. A minimum of two separate sets of skin specimens were prepared, imaged, and analyzed for each CER. Typical data are presented herein.

\section{Determination of IR extinction coefficients of CER [NP]-d3 I}

Chain perdeuteration of CER shifts the methylene stretching frequencies to a spectral region free from interference of endogenous skin vibrations. As IR spectra of skin sections were acquired in the transmission mode, Beer's law was applicable. To obtain extinction coefficients, CER [NP]-d31 was dissolved at known concentrations in chloroform:methanol 3:1 $(\mathrm{v} / \mathrm{v})$. A fixed path length $(16.4 \mu \mathrm{m}) \mathrm{IR}$ cell with $\mathrm{CaF}_{2}$ windows was used to measure the absorbance of each standard solution. IR spectra were acquired with a Nicolet 6700 spectrometer (Thermo Fisher Scientific, Waltham, MA, USA), co-adding 128 scans and using a spectral resolution of $2 \mathrm{~cm}^{-1}$.

The area of the asymmetric $\mathrm{CD}_{2}$ stretching $\left(\mathrm{v}_{\text {asym }} \mathrm{CD}_{2}\right)$ band (obtained using ISys software [v 5.0; Malvern Instruments Ltd, Malvern, UK]) was obtained as a function of molar concentration. Linear regression was used to find the best fit to the Beer's law plots. The extinction coefficient $\left(\mathrm{mol}^{-1}\right.$ $\mathrm{dm}^{3} \mathrm{~cm}^{-1}$ ) and correlation coefficient were 7,470 and 0.999, respectively. When calculating CER [NP]-d31 concentration, we assumed that the thickness (path length) of the skin section was $10 \mu \mathrm{m}$. The correlation coefficient demonstrates the high precision of using the asymmetric $\mathrm{CD}_{2}$ stretching band areas for calculating CER [NP]-d31 concentrations in skin.

\section{IR imaging data analysis}

Vibrational microspectroscopic images were generated from IR spectral data using ISys software (v 5.0; Malvern Instruments Ltd). Image planes of spectral parameters (integrated peak area or peak height) were produced after linear baselines were applied in spectral regions of interest. Image planes of CER concentrations were generated after using the extinction coefficient to estimate concentration. Figures were generated with SigmaPlot 2000 (SPSS Inc., Chicago, IL, USA).

\section{Results \\ Permeation of CER [NS]-d3 I}

Typical single-pixel IR spectra of CER [NS]-d31 at different depths in skin are shown in Figure 1A with bands of interest marked. Figure 1B highlights the $\mathrm{CD}_{2}$ stretching region $\left(2,234-2,064 \mathrm{~cm}^{-1}\right)$ at the same depths. By integrating the area under the asymmetric $\mathrm{CD}_{2}$ stretching band and converting to CER [NS]-d31 concentration utilizing Beer's law, we can quantitatively track the permeation of CER [NS]-d31 in skin. A high signal-to-noise ratio is evident in the spectra. 



Figure I (A) Typical single-pixel infrared spectra of N-palmitoyl-D-erythro-sphingosine (CER[NS]-d3I) at different depths in skin. (B) The $C_{2}$ stretching region $\left(2,234-2,064 \mathrm{~cm}^{-1}\right)$ is highlighted at the same depths in $\mathbf{A}$. This spectral region is baseline corrected.

The detection limit for CER [NS]-d31 is apparent in Figure $1 \mathrm{~B}$ at a depth $\sim 12.5 \mu \mathrm{m}$ from the skin surface (third spectrum from top) and is $3.5 \mathrm{mM}(\sim 1.3 \mathrm{wt} \%$ of SC lipid phase) where the peak area is approximately two times the noise level.

Figure 2 illustrates the spatial distribution and concentration of CER [NS]-d31 penetration in skin after a 24-hour incubation period. Figure 2A displays the visible micrographs of several skin sections whereas Figure 2B highlights IR images (of the same sections) of CER [NS]d31 concentration. Each IR image is comprised of 2,560 unique IR spectra (each pixel is observable in the image) with the CER [NS]-d31 concentration determined from the $\mathrm{CD}_{2}$ stretching region. CER [NS]-d31 appears to be heterogeneously distributed and localized in pockets close to the 


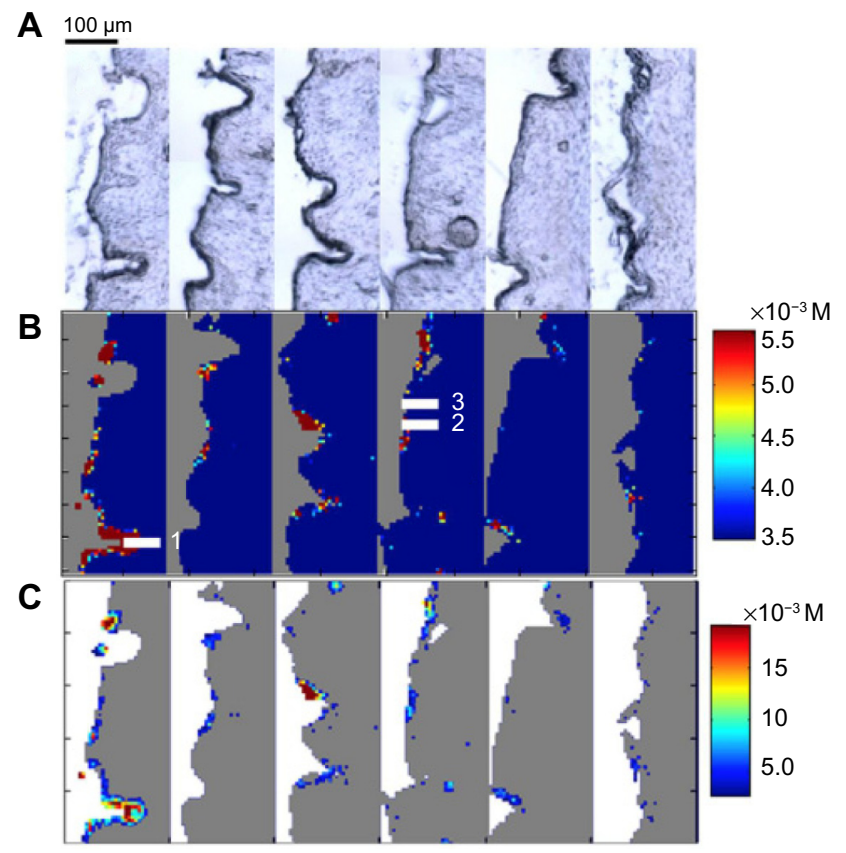

D



Figure 2 Spatial distribution and concentration of N-palmitoyl-D-erythro-sphingosine (CER [NS]-d3I) penetration in skin. (A) Visible micrographs of microtomed skin sections (stratum corneum [SC] on the left side of each section). (B) Infrared (IR) images (of the same sections) of CER [NS]-d3I concentration. The concentration range of 3.5-5.5 $\times 10^{-3} \mathrm{M}$ is shown to highlight the distribution of CER [NS]-d3I. The area outside the skin is shown in gray. (C) IR images of CER [NS]-d3I concentration above detection limit ( \pm I standard deviation). The concentration below the detection limit is shown in gray. The area outside the skin is shown in white. (D) Line plots of ceramide concentration and Amide II peak height were compared between glyph and non-glyph regions labeled in B; three to five adjacent lines of pixels are shown.

Notes: Blue lines and symbols: ceramide concentration; red lines and symbols: Amide II peak height. Magenta dash lines indicate the detection limit of $3.5 \times 10^{-3} \mathrm{M}$ of $\mathrm{CER}$. Scale bar is $100 \mu \mathrm{m}$.

Abbreviation: CER, ceramide.

skin surface. Figure $2 \mathrm{C}$ more clearly indicates the distribution of CER [NS]-d31 where regions below the detection limit are displayed in gray. Determining CER penetration into the skin is complicated by the nonplanar nature of the skin surface; glyphs and other features result in a complex topography. To more closely delineate CER penetration into the SC, as opposed to CER that remains on top of the SC, line plots of CER concentration and Amide II peak height were compared between glyph and non-glyph regions in Figure 2D. In the SC, Amide II band intensity predominantly arises from keratin. The surface of the skin is defined at the half-maximum Amide II peak height ( $\sim 0.2 \mathrm{AU}$ in the line plots). In the glyph set of plots, labeled "1" in Figure 2D, there is high CER concentration at $\sim 0-10 \mu \mathrm{m}$ from the image edge, in the region where the Amide II peak height is less than $0.2 \mathrm{AU}$. This demonstrates that there was a significant amount of CER [NS]-d31 within the glyph outside the SC that did not actually penetrate into the SC. There is some overlap, however, at $\sim 25 \mu \mathrm{m}$ from the image edge where the Amide
II intensity indicates the surface of the SC and where CER [NS]-d31 concentration is $\sim 0.008-0.015 \mathrm{M}$ within the SC. A similar phenomenon is also observed in line plots labeled "2" in Figure 2D in a non-glyph region with a relatively lower concentration of CER. The line plots labeled " 3 " were chosen at a place where CER was not observed in Figure 2B, and serve as a baseline for analysis of line plots 1 and 2. In summary, CER [NS]-d31 was heterogeneously distributed on top of the skin surface with small pockets of relatively low concentration penetrating $\sim 10-15 \mu \mathrm{m}$ into the SC.

Since we observed CER [NS]-d31 accumulating in glyph regions at relatively high concentrations, it was of interest to further explore these regions. Figure $3 \mathrm{~A}$ shows a visible image of a skin section with a deep glyph. Figure 3B displays the corresponding IR image of CER [NS]-d31 concentration, where a high CER concentration can be observed in the deep glyph region. Figure $3 \mathrm{C}$ and $\mathrm{D}$ focus on the $\mathrm{CER}$ concentration within the glyph area marked with a white box in Figure 3B. This concentration image has been rotated clockwise and enlarged in 


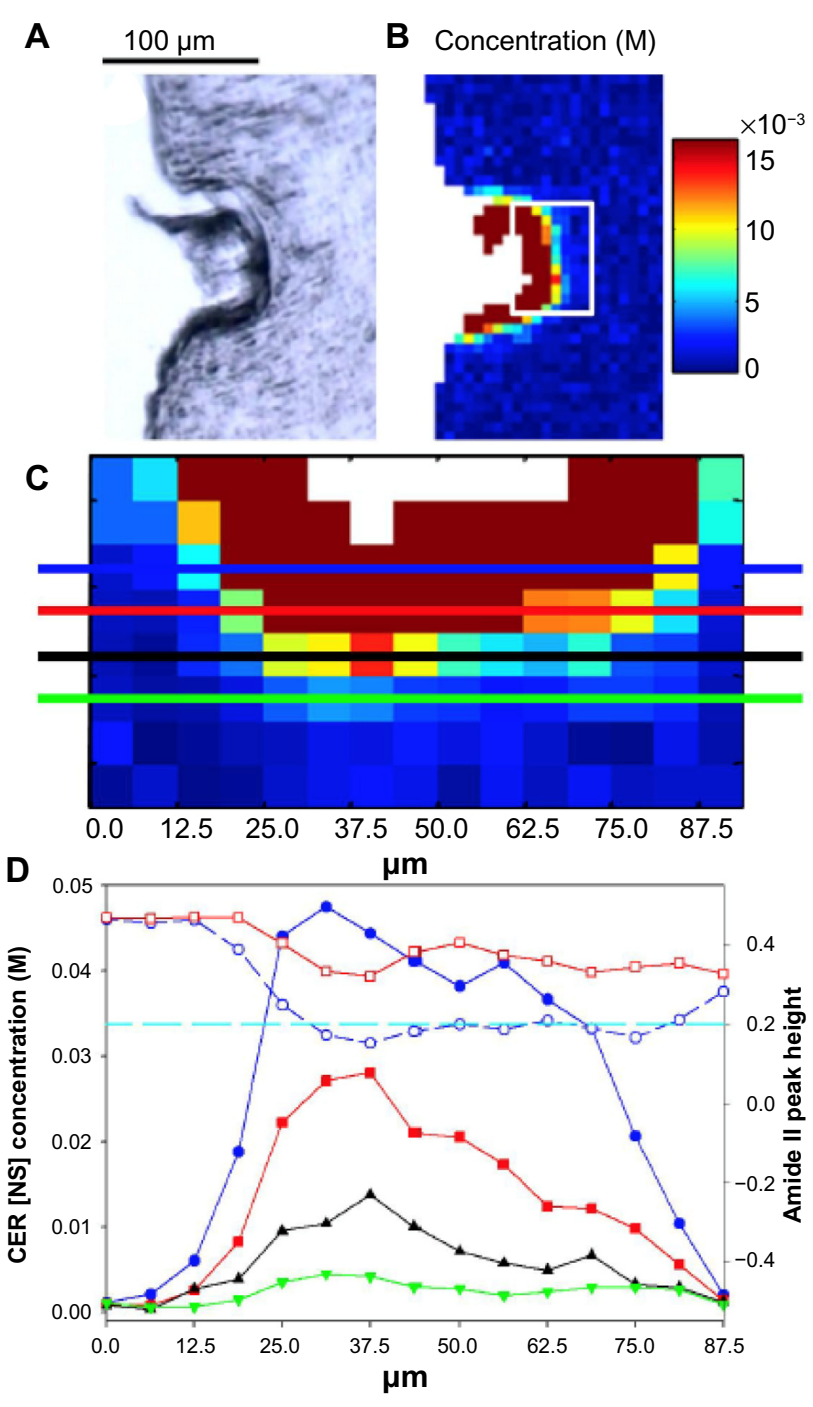

Figure 3 (A) Visible image of a skin section with a deep, broad glyph. (B) Infrared image of the $\mathrm{N}$-palmitoyl-D-erythro-sphingosine (CER [NS]-d3I) concentration profile for the same section. (C) Concentration image of the region marked with the white box in B, enlarged and rotated clockwise, along with color-coded lines highlighting the pixels from which the line plots in $\mathbf{D}$ originate. (D) Line plots of CER [NS]-d3I concentration and Amide II peak height as marked in C.

Notes: Closed symbol: CER [NS]-d3I concentration; open symbol: Amide II peak height. Cyan dashed line indicates the surface of the SC (Amide ll peak height $~ 0.2 \mathrm{AU}$ ). Scale bar is $100 \mu \mathrm{m}$.

Abbreviation: SC, stratum corneum

Figure 3C, while Figure 3D displays several line plots of CER concentration and Amide II peak height at the regions marked in 3C. The blue solid line plot indicates the CER concentration just at the surface of the SC (Amide II peak height $\sim 0.2 \mathrm{AU}$ ), whereas the other line plots display the CER concentration within the top $\sim 18 \mu \mathrm{m}$. CER concentrations can be observed to decrease rapidly from $\sim 20-28 \mathrm{mM}$ in the top $\sim 6 \mu \mathrm{m}$ to approximately the detection limit of $3.5 \mathrm{mM}$ at a depth of $18-20 \mu \mathrm{m}$.

\section{Permeation of CER [NP]-d3 I}

Penetration studies with CER [NP]-d31 (Figure 4B) show similar IR concentration profiles to CER [NS]-d31
( 4-10 mM) with visible images of the same CER [NP]-d31treated sections shown in Figure 4A. Results are shown for both 24- and 48-hour incubation periods. Figure 4C, with skin regions where CER concentration was below the detection limit masked in gray, depicts a heterogeneous spatial distribution of CER [NP]-d31 concentrations, localized in pockets on or within the SC surface and in deep glyph areas. The line plots shown in Figure 4D suggest that CER [NP]-d31 was heterogeneously distributed on the skin surface with small pockets at $\sim 8 \mathrm{mM}$ penetrating $\sim 10-15 \mu \mathrm{m}$ into SC. There was no significant difference between the 24- and 48-hour incubation times. Similar results were found comparing incubation times for CER [NS]-d31 (data not shown).

\section{OA penetration}

Two control experiments were conducted to compare the penetration and distribution of OA in skin with the observed limited CER penetration. In the first control experiment, skin samples were only treated with OA-d. In the second, a suspension of proteated CER [NS] in OA-d was topically applied. Figure 5A shows the visible images of control skin sections and Figure 5B depicts IR images of OA-d concentration and distribution by integrating the asymmetric $\mathrm{CD}_{2}$ stretching band. A more homogenous distribution of OA-d can be observed penetrating into the SC and VE, mainly concentrating in the SC. In contrast to the spatial distribution of both CER classes, OA-d is not concentrated in glyph regions (see glyph region in bottom of last image on right). Similar line plots of OA-d concentration and Amide II peak height are also compared in Figure 5C. One line plot is shown for each type of control experiment and both show a more homogenous distribution of OA-d throughout the SC and VE.

\section{Discussion}

Few previous studies have sought to quantify the transport of topically applied CER through healthy human skin. As far as we are aware, the majority of CER penetration studies have been conducted on acutely compromised skin in which recovery of SC barrier properties is monitored by transepidermal water loss (TEWL) and/or SC hydration by conductance measurements. ${ }^{6,7}$ For quantitative studies, tape stripping of the SC is commonly used along with highperformance liquid chromatography analysis. Increasing numbers of tapes are assumed to be removing deeper layers of skin. While subsequent tapes do in general remove deeper layers of the SC, tape stripping as a means of depth profiling is a source of significant ambiguity. ${ }^{26}$ One particular problem is determining what happens in glyphs. In tape stripping, 

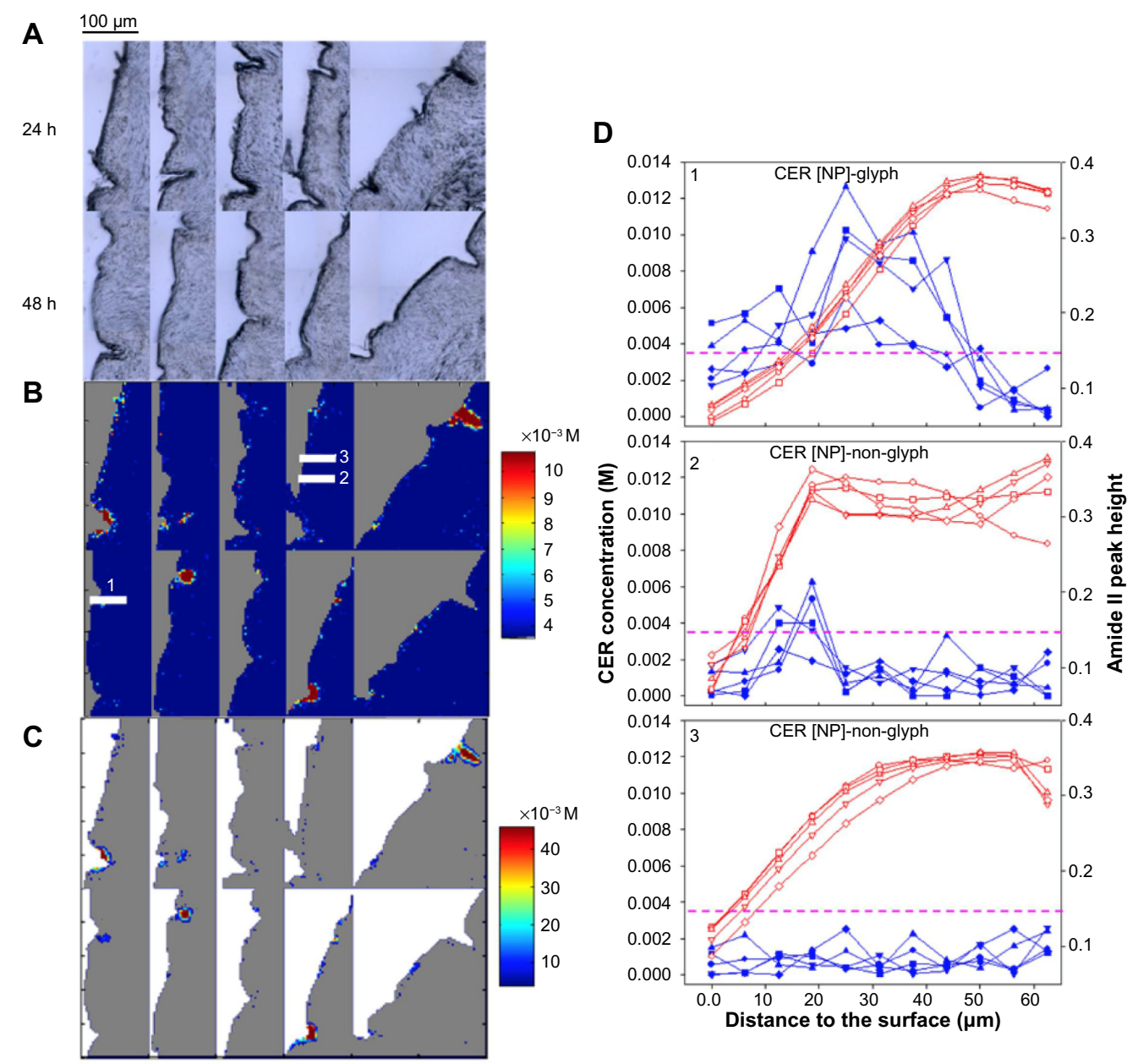

Figure 4 Spatial distribution and concentration of N-palmitoyl-D-erythro-phytosphingosine (CER [NP]-d3I) penetration in skin. (A) Visible micrographs of microtomed skin sections (stratum corneum on the left side of each section) for 24- and 48-hour incubation periods. (B) Infrared (IR) images (of the same sections) of CER [NP]-d3I concentration. The concentration range of 3.5-10.7 $\times 10^{-3} \mathrm{M}$ is shown to highlight the distribution of CER [NP]- $\mathrm{d} 3 \mathrm{I}$. The area outside the skin is shown in gray. (C) IR images of CER [NP]- $\mathrm{d} 3$ I concentration above the detection limit ( \pm I standard deviation). The concentration below the detection limit is shown in gray. Area outside the skin is shown in white. (D) Line plots of ceramide concentration and Amide II peak height were compared between glyph and non-glyph regions as labeled in $\mathbf{B}$ for five adjacent lines of pixels.

Notes: Blue: ceramide concentration; red: Amide II peak height. Magenta dashed lines indicate the detection limit of $3.5 \times 10^{-3} \mathrm{M}$. Scale bar is $100 \mu \mathrm{m}$.

material in glyphs can appear on tapes that predominantly sample layers deeper in the skin. As can be seen in Figures 2-4, topically applied CER appears to accumulate or be limited to the glyph regions. If tape stripping were done on these sections, the CER would appear on tapes thought to be sampling deeper within the skin. IR imaging provides CER concentration information with nonambiguous lateral and depth spatial information.

Imaging penetration using fluorescently labeled active agents also yields accurate lateral and depth spatial information; however, the fluorophore may alter penetration mechanisms. In a related report, Novotný et $\mathrm{al}^{8}$ examined the chain-length dependence of the penetration of fluorescently labeled CER [NS] in skin. The current results are reasonably consistent with this work for the longer chain CERs. Whereas Novotný et al reported that C6 CER penetrated to the $\mathrm{VE}$ and $\mathrm{C} 24$ resided only in the outer SC, the C16 CERs (both [NS] and [NP]) used in the study reported here were sporadically detected in the outer SC layers. In addition, although fluorescence detection is more sensitive than IR absorption, the application of Beer's law to IR images acquired in the transmission mode yields quantitative concentration values for the CERs in the SC glyph regions.

One of the goals of the current study was to demonstrate the potential of IR imaging to track the spatial distribution of the concentration of topically applied material in skin. The concentration of CERs and the pure OA enhancer were chosen to maximize CER penetration in skin. Further studies utilizing healthy skin and CERs with a variety of head groups, chain lengths, and formulations will help us evaluate the generality of these conclusions. 


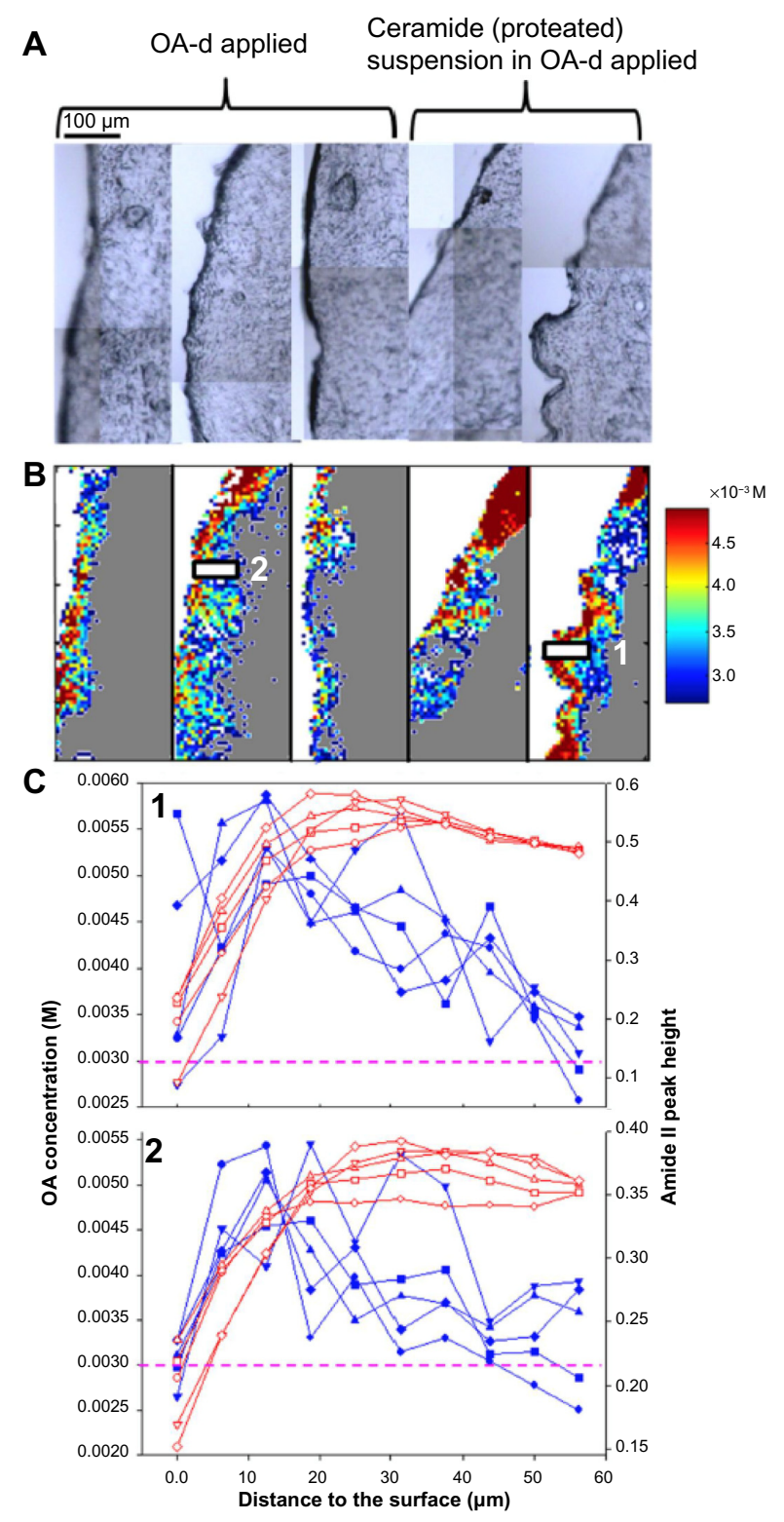

Figure 5 (A) Visible micrographs of control skin sections for two different treatments as labeled. (B) Infrared images of acyl chain perdeuterated oleic acid (OA-d) concentration and distribution in skin for the same sections. (C) Line plots of OA-d concentration and Amide II peak height were compared between the two controls as labeled in B (five adjacent lines of pixels).

Notes: Blue: OA-d concentration; red: Amide II peak height. Magenta dashed lines indicate the detection limit of $3.0 \times 10^{-3} \mathrm{M}$ of OA-d. Scale bar is $100 \mu \mathrm{m}$.

The current results also suggest that when OA is used as an enhancer, the topically applied CERs used herein remain predominantly within the glyphs. The recent study of Sahle et $\mathrm{al}^{9}$ demonstrated that various microemulsions can be used to control longer chain (C18) CER [NS] penetration across the SC. In that work, the CER concentration in upper and lower SC regions was quantified via tape stripping and highperformance liquid chromatography. In addition, uniformity in CER lateral distribution in the SC would seem to be important if CER is to incorporate into and enhance the structural barrier. Although Sahle et $\mathrm{al}^{9}$ observed a significantly higher concentration of exogenous CER in the SC, their approach, geared toward sensitivity, only delineated CER depth distribution, with no information about the lateral variations. Without an approach such as the one used here, it is difficult to tell whether barrier enhancement is indeed occurring or whether, for example, exogenous CER is accumulating in the glyphs.

\section{Acknowledgment}

This work was generously supported by Johnson \& Johnson Consumer Companies, Inc.

\section{Disclosure}

The authors declare no conflicts of interest in this work.

\section{References}

1. Huwiler A, Kolter T, Pfeilschifter J, Sandhoff K. Physiology and pathophysiology of sphingolipid metabolism and signaling. Biochim Biophys Acta. 2000;1485(2-3):63-99.

2. Bouwstra JA, Ponec M. The skin barrier in healthy and diseased state. Biochim Biophys Acta. 2006;1758(12):2080-2095.

3. Imokawa G, Abe A, Jin K, Higaki Y, Kawashima M, Hidano A. Decreased level of ceramides in stratum corneum of atopic dermatitis: an etiologic factor in atopic dry skin? J Invest Dermatol. 1991;96(4): 523-526.

4. Motta S, Monti M, Sesana S, Mellesi L, Ghidoni R, Caputo R. Abnormality of water barrier function in psoriasis. Role of ceramide fractions. Arch Dermatol. 1994;130(4):452-456.

5. van Smeden J, Janssens M, Gooris GS, Bouwstra JA. The important role of stratum corneum lipids for the cutaneous barrier function. Biochim Biophys Acta. 2014;1841(3):295-313.

6. Coderch L, López O, de la Maza A, Parra JL. Ceramides and skin function. Am J Clin Dermatol. 2003;4(2):107-129.

7. Feingold KR, Elias PM. Role of lipids in the formation and maintenance of the cutaneous permeability barrier. Biochim Biophys Acta. 2014; 1841(3):280-294.

8. Novotný J, Pospechova K, Hrabálek A, Cáp R, Vávrová K. Synthesis of fluorescent C24-ceramide: evidence for acyl chain length dependent differences in penetration of exogenous NBD-ceramides into human skin. Bioorg Med Chem Lett. 2009;19(24):6975-6977.

9. Sahle FF, Wohlrab J, Neubert RH. Controlled penetration of ceramides into and across the stratum corneum using various types of microemulsions and formulation associated toxicity studies. Eur J Pharm Biopharm. 2014;86(2):244-250.

10. Menon GK, Feingold KR, Elias PM. Lamellar body secretory response to barrier disruption. J Invest Dermatol. 1992;98(3):279-289.

11. Weerheim A, Ponec M. Determination of stratum corneum lipid profile by tape stripping in combination with high-performance thin-layer chromatography. Arch Dermatol Res. 2001;293(4):191-199.

12. Karande P, Mitragotri S. Enhancement of transdermal drug delivery via synergistic action of chemicals. Biochim Biophys Acta. 2009;1788(11): 2362-2373.

13. Liu CH, Chang FY, Hung DK. Terpene microemulsions for transdermal curcumin delivery: effects of terpenes and cosurfactants. Colloid Surf B Biointerfaces. 2011;82(1):63-70.

14. Deli G, Hatziantoniou S, Nikas Y, Demetzos C. Solid lipid nanoparticles and nanoemulsions containing ceramides: preparation and physicochemical characterization. J Liposome Res. 2009;19(3):180-188.

15. Müller RH, Shegokar R, Keck CM. 20 years of lipid nanoparticles (SLN and NLC): present state of development and industrial applications. Curr Drug Discov Technol. 2011;8(3):207-227. 
16. Shakeel F, Shafiq S, Haq N, Alanazi FK, Alsarra IA. Nanoemulsions as potential vehicles for transdermal and dermal delivery of hydrophobic compounds: an overview. Expert Opin Drug Deliv. 2012;9(8):953-974.

17. Cotte M, Dumas P, Besnard M, Tchoreloff P, Walter P. Synchrotron FT-IR microscopic study of chemical enhancers in transdermal drug delivery: example of fatty acids. J Control Release. 2004;97(2): 269-281.

18. Fernandez DC, Bhargava R, Hewitt SM, Levin IW. Infrared spectroscopic imaging for histopathologic recognition. Nat Biotechnol. 2005;23(4):469-474.

19. Mao G, Flach CR, Mendelsohn R, Walters RM. Imaging the distribution of sodium dodecyl sulfate in skin by confocal Raman and infrared microspectroscopy. Pharm Res. 2012;29(8):2189-2201.

20. Mendelsohn R, Flach CR, Moore DJ. Determination of molecular conformation and permeation in skin via IR spectroscopy, microscopy, and imaging. Biochim Biophys Acta. 2006;1758(7): 923-933.

21. Salzer R, Steiner G, Mantsch HH, Mansfield J, Lewis EN. Infrared and Raman imaging of biological and biomimetic samples. Fresenius J Anal Chem. 2000;366(6-7):712-716.
22. Xiao C, Moore DJ, Rerek ME, Flach CR, Mendelsohn R. Feasibility of tracking phospholipid permeation into skin using infrared and Raman microscopic imaging. J Invest Dermatol. 2005;124(3):622-632.

23. Gotter B, Faubel W, Neubert RH. FTIR microscopy and confocal Raman microscopy for studying lateral drug diffusion from a semisolid formulation. Eur J Pharm Biopharm. 2010;74(1):14-20.

24. Mack Correa MC, Mao G, Saad P, Flach CR, Mendelsohn R, Walters RM. Molecular interactions of plant oil components with stratum corneum lipids correlate with clinical measures of skin barrier function. Exp Dermatol. 2014;23(1):39-44.

25. Cosmetic Ingredient Review. Safety Assessment of Ceramides as Used in Cosmetics. Washington DC: Cosmetic Ingredient Review; 2015. Available from: www.cir-safety.org/sites/default/files/cerami032015rep. pdf. Accessed May 5, 2015.

26. van der Molen RG, Spies F, van 't Noordende JM, Boelsma E, Mommaas AM, Koerten HK. Tape stripping of human stratum corneum yields cell layers that originate from various depths because of furrows in the skin. Arch Dermatol Res. 1997;289(9):514-518.
Clinical, Cosmetic and Investigational Dermatology

\section{Publish your work in this journal}

Clinical, Cosmetic and Investigational Dermatology is an international, peer-reviewed, open access, online journal that focuses on the latest clinical and experimental research in all aspects of skin disease and cosmetic interventions. All areas of dermatology will be covered; contributions will be welcomed from all clinicians and

\section{Dovepress}

basic science researchers globally. This journal is indexed on CAS. The manuscript management system is completely online and includes a very quick and fair peer-review system, which is all easy to use. Visit http://www.dovepress.com/testimonials.php to read real quotes from published authors. 